\title{
Current-driven quantum criticality in itinerant electron ferromagnets
}

\author{
Aditi Mitra ${ }^{1}$ and Andrew J. Millis ${ }^{2}$ \\ ${ }^{1}$ Department of Physics, New York University, 4 Washington Place, New York, NY 10003 USA \\ ${ }^{2}$ Department of Physics, Columbia University, 538 W. 120th Street, New York, NY 10027 USA
}

(Dated: September 12, 2021)

\begin{abstract}
We determine the effect of an in-plane current flow on the critical properties of a $2 \mathrm{~d}$ itinerant electron system near a ferromagnetic -paramagnetic quantum critical point. We study a model in which a nonequilibrium steady state is established as a result of exchange of particles and energy with an underlying substrate. The current $\vec{j}$ gives rise not only to an effective temperature equal to the voltage drop over a distance of order the mean free path, but also to symmetry-breaking terms of the form $\vec{j} \cdot \vec{\nabla}$ in the effective action. The effect of the symmetry breaking on the fluctuational and critical properties is found to be small although (in agreement with previous results) if rotational degrees of freedom are important, the current can make the classically ordered state dynamically unstable.
\end{abstract}

PACS numbers: 73.23.-b,05.30.-d,71.10.-w,71.38.-k

One of the most intriguing issues in modern condensed matter physics is quantum criticality, the complex phenomena associated with phase changes driven by variation of Hamiltonian parameters such as pressure, magnetic field, or chemical composition in the limit as temperature $T \rightarrow 0$ [1, 2, 3, 4]. Quantum phase transitions differ from classical phase transitions because the noncommutativity of position and momentum in quantum mechanics implies that spatial and temporal fluctuations are coupled at a quantum critical point. While quantum phase transitions in equilibrium systems have been the subject of extensive study, phase transitions caused by nonequilibrium drives (for example an imposed current flow) have been much less extensively studied. Scaling theories and calculations have shown that one important effect of a departure from equilibrium is to produce decoherence effects which may be modeled as an effective temperature [5, 6, 7, 8, 9]. However, departures from equilibrium may lead to other effects [10]. In particular, a current drive breaks inversion symmetry, and it is well known that symmetry plays a crucial role in critical phenomena. In this paper we investigate the effect of a current drive on the critical properties of a low dimensional system near a ferromagnetic quantum critical point. We present a derivation from microscopics which reveals the inversion-symmetry-breaking terms, and we analyze their consequences.

The geometry that will be studied (shown schematically in Fig (1) is a very thin layer laid on top of a substrate with which the layer can exchange particles as well as energy. We take the layer to be a metal which is tuned to be near a ferromagnetic-paramagnetic quantum critical point. Departures from equilibrium and breaking of inversion symmetry are provided by current, $j$, flowing along the layer, while the coupling to the substrate allows the system to reach a nonequilibrium steady state. An earlier paper [5] studied a similar system but with nonequilibrium provided by current flow across the layer,

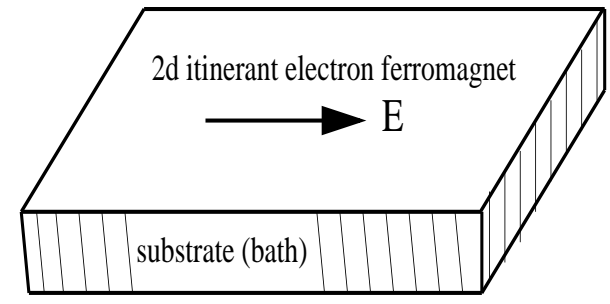

FIG. 1: A 2d itinerant electron ferromagnet driven out of equilibrium by application of an in-plane electric field. A steady state is reached via coupling to an underlying substrate.

so the issue of inversion symmetry breaking did not arise.

We now outline the relevant scales and summarize our major results. An electron in the layer will escape into the substrate after a typical time $\tau_{s c}$ determined by the strength of the coupling to the substrate and taken to be long compared to typical electronic times such as the inverse of the Fermi energy $E_{F}: E_{F} \tau_{s c}>>1$. The escape time implies a length $l_{s c}=v_{F} \tau_{s c}$ with $v_{F}$ the Fermi velocity in the layer. A current $j$ flowing in the layer implies an electric field $E=\rho j$. The departure from equilibrium produces decoherence whose consequences may be approximately parametrized by an effective temperature $T_{\text {eff }}[5,8]$ which in the present problem we find to be given by the voltage drop over an escape length;

$$
T_{e f f}=e E l_{s c}
$$

We present here a theory valid in the limit $T_{e f f} \tau_{s c}<<1$.

The new feature of the longitudinal current is a breaking of inversion symmetry. The magnitude of the symmetry breaking effect in the spin sector turns out to be related to the spin current $j_{M}=j_{\uparrow}-j_{\downarrow}$ induced by an applied electric field. In the model we study we find that for a magnetization density $M$ the spin current is $j_{M}=v_{D} M$ with magnetization drift velocity $v_{D}$ proportional both to the applied electric field [11, 12] and to 
deviations from particle-hole symmetry. The importance of particle-hole symmetry may be seen in a simple Boltzmann transport picture: the current $j_{\sigma}$ of electrons of spin $\sigma$ is $\vec{j}_{\sigma}=\int(d k) \vec{v}(k) n\left(\varepsilon_{k}-\mu_{\sigma} ; E\right)$ with $n$ a distribution function depending on the applied electric field, the electron energy, and the spin-dependent chemical potential. In a particle-hole symmetric situation, $v(k)$ is independent of $k$ and $\varepsilon_{k}=v|k|$, so a simple variable shift implies that $j_{\uparrow}=j_{\downarrow}$ implying $j_{M}=0$. In order of magnitude $v_{D} k_{F} \sim T_{\text {eff }}<<E_{F}$, so that the effects of inversion symmetry breaking turn out to be small.

We write a Keldysh path integral [13, 14], make a saddle point approximation followed by an expansion of the Keldysh action in fluctuations about the saddle point 5 , 15]. The resulting nonequilibrium Ginzburg-Landau theory is then treated by renormalization group [5, 16]. The Hamiltonian of the system is

$$
H=H_{\text {bath }}+H_{\text {layer }}+H_{\text {layer-bath }}
$$

where $H_{\text {layer }}$ is the interacting electron layer whose critical properties we are interested in, $H_{b a t h}$ describes the underlying substrate which will be assumed to be an electron gas which is always in thermodynamic equilibrium, while $H_{\text {layer-bath }}$ couples the two. Thus,

$$
\begin{aligned}
H_{\text {layer }} & =\sum_{\vec{p}_{\perp} \sigma} \epsilon_{\vec{p}_{\perp}-\frac{e}{\hbar c} \vec{A}} d_{\vec{p}_{\perp} \sigma}^{\dagger} d_{\vec{p}_{\perp} \sigma}+U \sum_{i} n_{i \uparrow} n_{i \downarrow}(3) \\
H_{\text {layer-bath }} & =\sum_{\vec{p}_{\perp}, \sigma}\left(t c_{\text {bath }, \sigma}^{\dagger} d_{\vec{p}_{\perp} \sigma}+\text { h.c. }\right)
\end{aligned}
$$

with $\sigma$ the spin label, $p_{\perp}$ the momentum within the layer which is a good quantum number, and $\vec{A}=-c \vec{E} t$. The only relevant features of $H_{b a t h}$ are its density of states, $N_{\text {bath }}$, which implies that $\tau_{s c}=\left(2 \pi N_{\text {bath }} t^{2}\right)^{-1}$ and its resistivity, which we take to be very high relative to that of the layer so we may couple the electric field only to the layer electrons.

The interaction term is treated via the usual HubbardStratonovich transformation, which involves introducing Hubbard-Stratonovich fields $m_{i \pm}$ in the time-ordered (denoted by -) and anti-time ordered (denoted by + ) Keldysh axes [5], choosing a quantization axis at each point in space and time [17] and introducing appropriate spin rotation matrices $\mathbf{R}_{ \pm}$. To simplify the notation we do not write these explicitly except where needed.

After integrating out the electronic degrees of freedom in the bath and layer we obtain the following Keldysh action for the classical $\left(m_{c l}=\frac{m_{-}+m_{+}}{2}\right)$ and quantum components $\left(m_{q}=\frac{m_{-}-m_{+}}{2}\right)$ of the auxiliary fields

$$
\begin{array}{r}
Z_{K}=\int \mathcal{D}\left[m_{q} m_{c l} \mathcal{D}[\Omega]\right] e^{-i \int d^{d} x d t 2 U m_{q} m_{c l}} \\
\times e^{\operatorname{Tr} \ln \left[i\left(G_{\sigma}^{-1}-U \sigma\left(\begin{array}{cc}
m_{c l} & m_{q} \\
m_{q} & m_{c l}
\end{array}\right)\right)\right]}
\end{array}
$$

where the Keldysh Greens function for the layer electrons

$$
G=\left(\begin{array}{cc}
G^{R} & G^{K} \\
0 & G^{A}
\end{array}\right)
$$

The effect of the nonequilibrium drive is expressed via the E-field dependence of $G$, which we now discuss. The solution of the Dyson equation for the retarded/advanced component of $G$ is

$$
G^{R / A}\left(\vec{p}, t_{1}, t_{2}\right)=\mp i \theta\left( \pm\left(t_{1}-t_{2}\right)\right) e^{-i \int_{t_{2}}^{t_{1}} d x \epsilon(\vec{p}+e \vec{E} x)} e^{-\frac{\left|t_{1}-t_{2}\right|}{2 \tau_{s c}}}
$$

Introducing the time difference $\tau=t_{1}-t_{2}$ and canonical momentum $\vec{k}=\vec{p}+e \vec{E} T,\left(T=\frac{t_{1}+t_{2}}{2}\right)$ we find that up to terms of order $(e E)^{2}\left(\tau_{s c}^{3} \partial^{2} \varepsilon_{k} /\left(\partial k^{2}\right)\right)$ in the exponential, the retarded/advanced components of the layer Green function $G$ take the equilibrium form

$$
G^{R / A}\left(p, t_{1}, t_{2} ; E\right) \rightarrow G^{R / A}(\vec{k}, \tau)=\mp i \theta( \pm \tau) e^{-i \epsilon_{k} \tau} e^{-\frac{|\tau|}{2 \tau_{s c}}}
$$

By introducing the lattice constant $a$ and noting that the fermi energy $E_{F} \sim v_{F} / a$, we may estimate the magnitude of the neglected terms as $\frac{\partial^{2} \epsilon / a^{2} \partial k^{2}}{E_{F}} \frac{\left(T_{e f f} \tau_{s c}\right)^{2}}{E_{F} \tau_{s c}}$ which is much less than unity in the limit of relevance here.

The Keldysh Green's function $G^{K}=G^{R} \cdot f_{K}-f_{K} \cdot G^{A}$ where the distribution function $f_{K}=1-2 f$ obeys

$$
\begin{aligned}
& i \frac{\partial f_{K}}{\partial t_{1}}+i \frac{\partial f_{K}}{\partial t_{2}}-\epsilon_{p-\frac{e}{\hbar c} A\left(t_{1}\right)} f_{K}+\epsilon_{p-\frac{e}{\hbar c} A\left(t_{2}\right)} f^{K} \\
& -\Sigma^{R} \cdot f_{K}+f_{K} \cdot \Sigma^{A}+\Sigma^{K}=0
\end{aligned}
$$

$\Sigma^{R}-\Sigma^{A}=-\frac{i}{\tau_{s c}}$ and $\Sigma^{K}=\left(\Sigma^{R}-\Sigma^{A}\right)(1-2 g), g$ being the distribution function of the substrate. Fourier transforming Eq. 9 with respect to the relative time $\tau=t_{1}-t_{2}$, changing variables to the canonical momentum $\vec{k}=\vec{p}+e \vec{E} T$ and expanding in $E$ one finds that the distribution function at steady state obeys,

$$
\begin{aligned}
& e \vec{E} \cdot \frac{\partial f}{\partial \vec{k}}+\frac{\partial f}{\partial \omega}\left(e \vec{E} \cdot \frac{\partial \epsilon_{k}}{\partial \vec{k}}\right)+\frac{1}{24} \frac{\partial^{3} f}{\partial \omega^{3}}\left(e \vec{E} \cdot \frac{\partial}{\partial \vec{k}}\right)^{3} \epsilon_{k} \ldots \\
& =\frac{1}{\tau_{s c}}[-f+g]
\end{aligned}
$$

The usual quasiclassical arguments [18] imply that the first term in Eq 10 is negligible while in the the weak field limit the third term may be dropped. With these simplifications we find $f=f^{s}+f^{a}$ where

$$
\begin{aligned}
f_{k, x}^{s} & =\theta(-x)+\frac{\operatorname{sign}(x)}{2} e^{-\frac{|x|}{\sqrt{\left(e \vec{E} \cdot \vec{v}_{k} \tau_{s c}\right)^{2}}}} \\
f_{k, x}^{a} & =\frac{\left(e \vec{E} \cdot \vec{v}_{k} \tau_{s c}\right)}{2 \sqrt{\left(e \vec{E} \cdot \vec{v}_{k} \tau_{s c}\right)^{2}}} e^{-\frac{|x|}{\sqrt{\left(e \vec{E} \cdot \vec{v}_{k} \tau_{s c}\right)^{2}}}}
\end{aligned}
$$

where $x=\omega-\mu$ and $v_{k}=\partial \varepsilon_{k} / \partial k$. Substitution of Eqs 1112 into Eq10 then shows that the neglect of the third 
term in Eq10 is justified at weak layer-substrate coupling $\left(\frac{\partial^{3} \epsilon_{k}}{E_{F} a^{3} \partial k^{3}} \ll\left(E_{F} \tau_{s c}\right)^{2}\right)$ while the first term is negligible in the weak field limit $T_{\text {eff }} \ll E_{F}^{2} /\left(\frac{\partial^{2} \epsilon_{k}}{a^{2} \partial k^{2}}\right)$.

The quantum critical analysis proceeds [5, 14] by finding a saddle point with $m_{q}=0$ and an $m_{c l}$ that obeys

$$
2 i U m_{c l}=-U T r\left[\left(G_{\sigma}^{-1}-\sigma U m_{c l}\right)^{-1} \sigma \tau_{x}\right]
$$

where $\tau$ are Pauli matrices in Keldysh space, and then expanding about the saddle point and using renormalization group methods to treat the resulting action. In the disordered phase the saddle point is $m_{c l}=m_{q}=0$ and by expanding the Trln in Eq. 5 we obtain

$$
Z_{K}=\int \mathcal{D}\left[m_{q} m_{c l}\right] e^{-i \int d^{d} x d t 2 U m_{q} m_{c l}} e^{S_{K}^{2}+S_{K}^{3}+S_{K}^{4}+. .}
$$

where

$$
S_{K}^{2}=-i U \int \frac{d^{d} q}{(2 \pi)^{d}} \frac{d \Omega}{2 \pi}\left[m_{q}^{*} m_{c l} \Pi^{R}+\text { c.c. }\right]+m_{q} m_{q}^{*}\left[\Pi_{K}(15)\right.
$$

involves polarization bubbles $\Pi_{R / A / K}$ computed in terms of the $G$. For the Ising case $S_{K}^{3}=0$, while $S_{K}^{4}=$ $\sum_{i=1 \ldots 4} u_{i} m_{q}^{i} m_{c l}^{4-i}$. We have used Eqs 8, 11 and 12 to compute the $\Pi$ and $u_{i}[5]$. Expanding in $q, \Omega$, distance from criticality $\delta$ and $T_{\text {eff }} \tau_{s c}$ we find

$$
U \Pi^{R}(q, \Omega)=\delta+A q^{2}-i \tau_{s c}\left(\Omega-\vec{v}_{D} \cdot \vec{q}\right)
$$

The $i\left(\Omega-\vec{v}_{D} \cdot \vec{q}\right) \tau_{s c}$ factor expresses the non-conserved dynamics induced by the escape of electrons from the layer into the substrate; the form of this term shows that the fluctuations drift at the magnetization drift velocity $v_{D}$ which in the approximations considered here is

$$
\vec{v}_{D}=e \vec{E} \tau_{s c} \frac{1}{N_{0}} \int \frac{d^{d} k}{(2 \pi)^{d}} \delta\left(\epsilon_{k}-\mu\right) \frac{\partial^{2} \epsilon_{k}}{\partial k^{2}}
$$

where $N_{0}=\int \frac{d^{d} k}{(2 \pi)^{d}} \delta\left(\epsilon_{k}-\mu\right)$. The drift term was missed in previous work [6]. To estimate its order of magnitude we note that Eq17involves the scale $T_{\text {eff }}$ and bare quantities. Because one may regard the dimension of velocity as $[$ Energy $\times$ Length $]$ and the only available length is the fermi wave vector, we estimate $v_{D}=T_{e f f} / k_{F}$.

The Keldysh polarization bubble contains information about decoherence and noise. In contrast to $\Pi^{R / A}, \Pi^{K}$ is found to depend on $\Omega$ only because the noise arises from coupling to the substrate and does not drift in the presence of a current in the layer. We find in $2 \mathrm{~d}$

$$
\begin{aligned}
\Pi_{2 d}^{K}(\Omega) & =-2 i \tau_{s c} \\
& {\left[|\Omega|+T_{\text {eff }} \int_{-\pi}^{\pi} \frac{d \phi}{2 \pi}|\cos \phi| e^{-\frac{|\Omega|}{\left(T_{e f f}|\cos \phi|\right)}}\right] }
\end{aligned}
$$

and for a 1d system,

$$
\Pi_{1 d}^{K}(\Omega)=-2 i \tau_{s c}\left[|\Omega|+T_{e f f} e^{-\frac{|\Omega|}{T_{e f f}}}\right]
$$

Eqs 18 and 19 show that the scale $T_{\text {eff }}$ is similar to a temperature in that $\Pi^{K}$ is proportional to $\Omega$ for $\Omega>T_{\text {eff }}$ but is proportional to $T_{\text {eff }}$ for $\Omega<T_{\text {eff }}$. An electric field induced effective temperature was previously identified in [5], where the current flow across the layer controlled the decoherence, and in [6] where the equivalent of $\tau_{s c}$ was argued to arise from electron-magnon scattering which itself depended on $T_{e f f}$, leading to a nonlinear relation between $T_{\text {eff }}$ and $E$.

We now present a renormalization group treatment of Eq. 14. The theory involves a momentum cutoff $\Lambda \sim k_{F}$ and we define the energy unit to be $v_{F} \Lambda$ which we assume to be $>>T_{\text {eff }}$. We also assume $v_{D}<v_{F}$ and $u, \delta<<1$. We integrate out momenta in a small range $\Lambda$ to $\Lambda e^{-l}$ and simultaneously integrate out all frequencies from $-\infty$ to $\infty$ (this procedure makes the preservation of causality manifest [5]). We then rescale momentum $q \rightarrow q e^{-l}$, the frequency $\omega \rightarrow \omega e^{-z l}$, and the fields $m_{i} \rightarrow m_{i} e^{\alpha l}$, so as to keep the second and third terms in Eq. 16 invariant, implying dynamical exponent $z=2$. We have

$$
\begin{aligned}
\frac{d T_{\text {eff }}}{d l} & =2 T_{\text {eff }} \\
\frac{d \delta}{d l} & =2 \delta+C_{1} u_{1} \\
\frac{d v_{D}}{d l} & =v_{D} \\
\frac{d u_{i}}{d l} & =[2-d] u_{i}+\mathcal{O}\left(u_{k} u_{l}\right)
\end{aligned}
$$

Here $C_{1}=\lim _{v_{F} \Lambda / T_{\text {eff }} \rightarrow \infty} \int_{-\infty}^{\infty} \frac{d \omega}{2 \pi} \frac{U \Pi_{K}\left(\omega, T_{\text {eff }}\right)}{U \Pi^{R}(q=\Lambda, \omega) U \Pi^{A}(q=\Lambda, \omega)}$.

Solving in dimension $d=2$ gives [2, 5] $T(l)=T_{\text {eff }}^{0} e^{2 l}$, $v_{D}(l)=v_{D}^{0} e^{l}, \delta(l)=r_{0} e^{2 l}$ and $u_{1}(l)=2 \pi^{2} /(9 l)$ after initial transients have decayed. Here the superscript 0 denotes initial values and $r_{0}$ is related to $\delta_{0}$ and $u$ as described in [2]. The most interesting regime is the quantum critical/renormalized classical one occurring for $T_{\text {eff }}^{0}>\left|r_{0}\right|$. In this case, at the scale $l_{T} \approx \ln \sqrt{1 / T_{\text {eff }}^{0}}$ a crossover to classical scaling occurs. Eq. 22 shows that the drift term remains small $\left(v_{D}\left(l_{T}\right) \Lambda<<T\left(l_{T}\right) \sim 1\right)$ so that up to corrections of order $\sqrt{T_{\text {eff }}^{0} / E_{F}}$ the drift does not affect the scaling. The treatment of the crossover to the classical regime follows the procedure given in [5] leading to a long wavelength theory described by the Halperin-Hohenberg model A dynamics, but with an extra $\vec{j} \cdot \vec{\nabla}$ coupling to the order-parameter which represents current induced drift. Restoring physical units,

$$
\begin{aligned}
& \tau_{s c}\left(\frac{\partial}{\partial t}-\vec{v}_{D} \cdot \vec{\nabla}\right) m_{c l}(x, t) \\
& =-\left[r-A \nabla^{2}+\frac{2 \pi^{2} m_{c l}^{2}}{9 \ln \sqrt{1 / T_{\text {eff }}^{0}}}\right] m_{c l}+\xi
\end{aligned}
$$


where

$$
\left\langle\xi(x, t) \xi\left(x^{\prime}, t^{\prime}\right)\right\rangle=\left(\frac{2 \tau_{s c} T_{e f f}}{\pi}\right) \delta\left(t-t^{\prime}\right) \delta\left(x-x^{\prime}\right)
$$

The delta-correlated nature of the noise means that the low energy theory is Galilean invariant so that the drift may be eliminated by the transformation $x \rightarrow x-v_{D} t$.

We now turn to the case of a Heisenberg magnet which may be treated along very similar lines except that we require a vector Hubbard-Stratonovich field [17] which we denote by $\vec{\Delta}$. The physics is very similar to the Ising case except for fluctuations about the ordered state. We concentrate on this in what follows. Expanding around $\vec{\Delta}_{c l}=\left\langle m_{c l}\right\rangle \hat{z}, \vec{\Delta}_{q}=0$ where $\left\langle m_{c l}\right\rangle$ is given by Eq 13, one obtains the Landau-Lifshitz-Gilbert equation of motion for the transverse spin wave fluctuations:

$$
\left(A \mathbf{1}+i B \tau_{y}\right)\left(\begin{array}{c}
\Delta_{c l}^{x} \\
\Delta_{c l}^{y}
\end{array}\right)=\xi
$$

with $A=\alpha_{x x}\left[\frac{\partial}{\partial t}-\vec{v}_{D x x} \cdot \vec{\nabla}\right]-C \nabla^{2}$ and $B=$ $\alpha_{x y}\left[\frac{\partial}{\partial t}-\vec{v}_{D x y} \cdot \vec{\nabla}\right]-D \nabla^{2}$ where $\xi$ is the white noise defined in Eq. 25. The spin-wave correlation function obtained from Eq. 26 may be computed by standard methods and is found to evolve in time as $e^{-F\left|t_{1}-t_{2}\right|}$ with

$$
\operatorname{Re}[F]=\frac{\left(\alpha_{x x} C+\alpha_{x y} D\right) q^{2}+\alpha_{x x} \alpha_{x y}\left(\vec{v}_{D x x}-\vec{v}_{D x y}\right) \cdot \vec{q}_{27)}}{\alpha_{x x}^{2}+\alpha_{x y}^{2}}
$$

showing that a dynamical instability signaled by $F^{R}<0$ occurs if even an infinitesimally small current is applied to a magnet of Heisenberg symmetry, provided $\vec{v}_{D x x} \neq \vec{v}_{D x y}$. For $v_{D x x}=v_{D x y}$, the $\vec{v}_{D} \cdot \vec{q}$ term may be eliminated via a Galilean transformation and the current does not drive a spin-wave instability. $v_{D x x}-v_{D x y}$ is a non-universal quantity. In the model defined by Eqs 34] $v_{D x x}$ differs from $v_{D x y}$ only in the presence of energy or momentum dependent scattering rates.

A derivation from microscopics of the current induced drift within a linear response treatment was presented in [19, 20] for models with impurity scattering. Our treatment here differs in two ways, one is that we consider a clean system where the dominant scattering mechanism is via an inelastic coupling to an external reservoir. Secondly we go beyond linear response, which allows us to capture the effect of current induced noise. Ref [21] showed that in the absence of noise Eq 26 has chaotic dynamics in the instability regime; the modifications of their results in the presence of noise and critical fluctuations is an interesting open question.

In summary we have derived from a fundamental theory the effect of an in-plane current on a magnetic quantum critical point. The current flow causes the critical fluctuations to drift with a velocity related to the spin current; however for Ising symmetry we find that in the regime where critical fluctuations are important the theory is effectively Galilean-invariant so the drift may be eliminated by an appropriate transformation. In models with rotational invariance the current leads to a spin wave instability whose critical behavior might be interesting to study further; however in the models we have studied the symmetry breaking effects are numerically small so the main effect of the departure from equilibrium is an effective temperature, proportional to the voltage drop over a distance of order of the mean free path. Extension of these results to the superconducting case where the direct coupling between the order-parameter and the current can give rise to other nonequilibrium effects besides noise is currently in progress.

Acknowledgements: The authors gratefully acknowledge useful discussions with Igor Aleiner. This work was supported by NSF-DMR-0705584.

[1] J. A. Hertz, Phys. Rev. B, 14, 1165 (1976).

[2] A. J. Millis, Phys. Rev. B, 48, 7183 (1993).

[3] S. L. Sondhi, S. M. Girvin, J. P. Carini and D. Shahar, Rev. Mod. Phys., 69, 315 (1997).

[4] S. Sachdev, Quantum Phase Transitions, Cambridge University Press, Cambridge, England (1999).

[5] Aditi Mitra, S. Takei, Y. B. Kim and A. J. Millis, Phys. Rev. Lett., 97, 236808 (2006).

[6] P. M. Hogan and A. G. Green, cond-mat/0607522

[7] D. Dalidovich and P. Phillips, Phys. Rev. Lett., 93, 027004 (2004).

[8] A. G. Green and S. L. Sondhi, Phys. Rev. Lett., 95, 267001 (2005).

[9] S. Takei and Y. B. Kim, arXiv:0712.1043.

[10] D. E. Feldman, Phys. Rev. Lett., 95, 177201 (2005).

[11] Ya. B. Bazaliy, B. A. Jones, and Shou-Cheng Zhang, Phys. Rev. B, 57 , 3213 (1998).

[12] J. Fernandez-Rossier, M. Braun, A. S. Núñez and A. H. MacDonald, Phys. Rev. B, 69, 174412 (2004).

[13] L. V. Keldysh, Zh. Eksp. Teor. Fiz, 47, 1515 (1964) [Sov. Phys. JETP, 20, 1018 (1965)].

[14] Alex Kamenev, cond-mat/0412296

[15] A. Mitra, I. Aleiner and A. J. Millis, Phys. Rev. Lett., 94, 076404 (2005).

[16] Aditi Mitra and A. J. Millis, Phys. Rev. B, 76, 085342, 2007.

[17] H. J. Schulz, Phys. Rev. Lett. 65, 2462 (1990)

[18] J. Rammer and H. Smith, Rev. Mod. Phys., 58, 323 (1986).

[19] R. A. Duine, A. S. Núñez, Jairo Sinova, and A. H. MacDonald, Phys. Rev. B, 75, 214420 (2007).

[20] G. Tatara, H. Kohno, J. Shibata, Y. Lemaho and K. Lee, J. Phys. Soc, Japan, 76, 054707 (2007).

[21] Z. Li, J. He and S. Zhang, Journal of Applied Physics, 97, 10C703 (2005). 